\title{
CDX-1/CDX-2 Expression Is a Favorable Prognostic Factor in Epstein-Barr Virus-Negative, Mismatch Repair-Proficient Advanced Gastric Cancers
}

\author{
Kyeongmin Kim¹, Songmi Noh², Jae-Ho Cheong ${ }^{3}$, and Hyunki Kim ${ }^{1}$ \\ 1Department of Pathology, Yonsei University College of Medicine, ${ }^{2}$ Department of Pathology, CHA Gangnam Medical Center, CHA \\ University, and ${ }^{3}$ Department of Surgery, Yonsei University College of Medicine, Seoul, Korea
}

\section{Article Info}

Received June 30, 2020

Revised September 25, 2020

Accepted October 5, 2020

Published online July 28, 2021

Corresponding Author

Hyunki Kim

ORCID https://orcid.org/0000-0003-2292-5584

E-mail KIMHYUNKI@yuhs.ac
Background/Aims: Caudal type homeobox (CDX)-1 and -2 are reportedly involved in the development and progression of gastric cancer (GC). Although there are several reports on the prognostic significance of CDX-2 expression in GC, it remains controversial. In this study, we sought to validate the prognostic value of CDX-1 and -2 expression according to the histologic and molecular subtypes of GC.

Methods: In total, 1,158 cases of advanced GC were investigated using immunohistochemical staining and tissue microarrays for CDX-1 and -2 expression, and survival analysis was performed according to different histological and molecular subtypes.

Results: Of the 915 GCs with CDX-1 expression, 163 (17.8\%) were Epstein-Barr virus (EBV)positive or mismatch repair deficient (MMR-d), and the remaining $752(82.2 \%)$ were EBV-negative or MMR-proficient (MMR-p). Of the 1,008 GCs with CDX-2 expression, 177 (17.5\%) were EBV-positive or MMR-d, and the remaining 831 (82.5\%) were EBV-negative or MMR-p. In the EBV-positive and MMR-d groups, CDX expression had no relationship with patient outcomes. In the EBV-negative and MMR-p groups, 404 (53.7\%) and 523 (62.9\%) samples were positive for CDX-1 and CDX-2 expression, respectively. Survival analysis demonstrated that CDX-1 and CDX-2 expression in all patients was correlated with favorable outcomes in terms of overall survival (multivariate analysis; $p=0.018$ and $p=0.028$, respectively). In the subgroup analysis, $C D X-1$ expression and CDX-2 expression were associated with favorable outcomes in EBV-negative and MMR-p intestinal $(p=0.015$ and $p=0.010)$, and mixed and diffuse-type $(p=0.019$ and $p=0.042)$ GCs, respectively.

Conclusions: The expression of CDX-1 and CDX-2 is a favorable prognostic factor in EBVnegative, MMR-p advanced GC. (Gut Liver 2021;15:694-704)

Key Words: CDX-1; CDX-2; Gastric cancer; Prognosis

\section{INTRODUCTION}

Caudal type homeobox (CDX)-1 and -2 , members of the caudal-related homeobox gene family, are intestinespecific transcriptional factors related to the proliferation and differentiation of intestinal epithelial cells. The role of CDX-1 and CDX-2 in intestinal metaplasia in the stomach has been demonstrated in a transgenic mouse model. ${ }^{1,2}$ CDX-2 expression is also known to be associated with intestinal metaplasia grade and to play an important role in the progression of neoplastic change. ${ }^{3}$ Moreover, research has also revealed that CDXs are involved in the development and progression of gastric cancer (GC).$^{4-8}$ Accordingly, several studies have investigated the prognostic significance of CDX expression in GCs and suggested that CDX-2 expression is correlated with good prognostic features in GC. ${ }^{9}$ For example, Ru et al. ${ }^{10}$ demonstrated that the expression of CDX-2 in GC is associated with better differentiation and a lower rate of lymph node metastasis. However, Ge et al. ${ }^{11}$ and Roessler et al. ${ }^{12}$ found that CDX-2 expression 
was associated with reduced cell proliferation rates and that CDX-2 expression decreased progressively with the depth of tumor invasion and progress of the advanced stages of GC. Moreover, Xiao et al. ${ }^{13}$ suggested that there was no significant correlation between CDX-2 expression and prognostic clinicopathological parameters. A metaanalysis indicated that CDX-2 expression was significantly associated with a lower clinical stage and higher 5-year survival rate. ${ }^{4}$ However, the number of studies that were analyzed for prognostic significance in the meta-analysis study totaled only four, and the total number of cases was 475. Thus, the previous meta-analysis study may not have had a large enough number of cases with which to evaluate the significance of CDX-2 expression in GC. Therefore, the prognostic significance of CDX-2 expression in GC remains to be elucidated.

GC is a markedly heterogeneous disease in terms of histologic features and molecular characteristics, resulting in a lack of effective chemotherapeutic agents, and the prognostic value of a biomarker could vary depending on the cancer stage and histologic and molecular subtypes. ${ }^{14}$ GCs can be divided into four molecular subgroups: (1) Epstein-Barr virus (EBV)-positive tumors, which occur most frequently in the proximal stomach, including the cardia, fundus and body, and display PIK3CA mutation, extreme DNA hypermethylation, and amplification of JAK2, $P D$ $L 1$, and $P D-L 2$; (2) microsatellite unstable tumors, which show elevated mutation rates throughout the genome due to mismatch repair (MMR) deficiency, in addition to distinct clinicopathologic features and better prognoses; ${ }^{15}$ (3) genomically stable GCs, which are related to signet ring cell carcinoma or diffuse histology and frequent mutations in $\mathrm{CDH} 1$ and $\mathrm{RHOA}$ genes; and (4) chromosomal instability GCs, which show marked aneuploidy and frequent amplification of receptor tyrosine kinases, such as HER2 (receptor tyrosine-protein kinase erbB-2), EGER (epidermal growth factor receptor), and C-MET. ${ }^{16}$

While it is well-known that CDX-1 is related to intestinal metaplasia in the stomach, data on CDX-1 expression as a prognostic factor in GC are lacking. Recently, we found that, as a marker, CDX-1 was related to benefits from adjuvant chemotherapy after surgery in patients with stage II-III GC using data from five cohorts of publicly available transcriptome profiling data (total, $\mathrm{n}=1,259$ tumor samples). ${ }^{17}$ As this result was derived from RNA expression, it may be difficult to apply in daily practice in the real world.

Therefore, in this study, we attempted to validate and apply the CDX-1 results of our previous study in a different large cohort using an immunohistochemical (IHC) approach, which is a feasible modality in daily practice, and to analyze the prognostic significance of CDX-1 and
CDX-2 expression according to the different histologic and molecular subtypes of GC.

\section{MATERIALS AND METHODS}

\section{Patients and tissue collection}

A total of 1,158 patients with advanced GC (760 males and 398 females) who underwent gastrectomy with D2 lymph node dissection at Yonsei University College of Medicine between January 2000 and December 2003 were consecutively enrolled. Patients who had undergone preoperative chemotherapy or radiotherapy and those who had undergone surgery for recurrent cancer were excluded. The mean age of the patients was 56.8 years (range, 25 to 88 years), and the mean follow-up duration was 57.7 months (range, 2.0 to 109.4 months). Patient clinical information and survival data were obtained from medical records and the Korean Central Cancer Registry. This study was approved by the Institutional Review Board of Yonsei University College of Medicine (IRB number: 4-2014-0668).

\section{Tissue microarray construction and IHC staining}

Two cores of tumor tissue ( $3 \mathrm{~mm}$ in diameter) were punched out from individual formalin-fixed and paraffinembedded tumor blocks and arrayed in a new tissue microarray (TMA) block. A core of adjacent non-neoplastic mucosa was arrayed in each TMA block as a landmark and internal control. The non-neoplastic mucosa core was sampled from the adjacent mucosa in the tumor block. Sections ( $4-\mu \mathrm{m}$ thick) from each TMA block were prepared for IHC staining. Hematoxylin and eosin and cytokeratin IHC staining were performed to confirm the presence of tumor cells. IHC staining with antibodies for CDX-1 (1:100; Abcam, Cambridge, UK) and CDX-2 (1:400; Cell Marque, Rocklin, CA, USA) was carried out using a Ventana Discovery XT automated staining system (Ventana Medical Systems, Tucson, AZ, USA).

The stained TMA slides were independently reviewed by two pathologists (H.K. and K.K.) who were unaware of any patient medical information. The expression patterns of CDX-1 and CDX-2 were categorized into three groups based on the most strongly expressed area in the two cores: (1) strong (2+), in which staining was nuclear and stronger than that of the normal gastric mucosa; (2) moderate and weak $(1+)$, in which staining was nuclear with a similar or weaker intensity than that of the normal gastric mucosa; and (3) absent staining (negative), in which no tumor cells were expressed. We interpreted the IHC staining result as positive for $1+$ and $2+$ patterns, and negative for an absent staining (negative) pattern. 
Table 1. Clinicopathologic Characteristics of Advanced Gastric Cancers According to the Expression Status of CDX-1 and CDX-2

\begin{tabular}{|c|c|c|c|c|c|c|c|c|}
\hline \multirow{2}{*}{ Variable } & \multicolumn{4}{|c|}{ CDX-1 } & \multicolumn{4}{|c|}{ CDX-2 } \\
\hline & Cases & Positive & Negative & $\mathrm{p}$-value & Cases & Positive & Negative & p-value \\
\hline Total & 938 & 471 & 467 & & 1,027 & 591 & 436 & \\
\hline Age, yr & & $57.3 \pm 12.3$ & $57.1 \pm 11.8$ & 0.779 & & $56.6 \pm 12.6$ & $56.9 \pm 11.9$ & 0.692 \\
\hline Overall survival, mo & & $82.8 \pm 55.5$ & $74.5 \pm 54.6$ & 0.022 & & $80.3 \pm 54.6$ & $74.7 \pm 55.9$ & 0.112 \\
\hline Disease-free survival, mo & & $77.1 \pm 58.8$ & $68.6 \pm 57.4$ & 0.027 & & $73.9 \pm 57.9$ & $69.9 \pm 58.7$ & 0.287 \\
\hline Sex & & & & 0.215 & & & & 0.947 \\
\hline Male & $618(65.9)$ & 301 (63.9) & $317(67.9)$ & & $672(65.4)$ & $386(65.3)$ & $286(65.6)$ & \\
\hline Female & $320(34.1)$ & $170(36.1)$ & $150(32.1)$ & & $355(34.6)$ & 205 (34.7) & $150(34.4)$ & \\
\hline Differentiation & & & & 0.063 & & & & 0.025 \\
\hline Differentiated & 277 (53.8) & $126(26.8)$ & 151 (32.3) & & 293 (28.5) & 185 (31.3) & 108 (24.8) & \\
\hline Undifferentiated & $661(46.2)$ & 345 (73.2) & $316(67.7)$ & & 734 (71.5) & 406 (68.7) & $328(75.2)$ & \\
\hline Lauren classification & & & & $<0.001$ & & & & 0.95 \\
\hline Intestinal or mixed & 502 (53.5) & $215(45.6)$ & 287 (61.5) & & $538(52.4)$ & 309 (52.3) & 229 (52.5) & \\
\hline Diffuse & $436(46.5)$ & $256(54.4)$ & 180 (38.5) & & $489(47.6)$ & $282(47.7)$ & 207 (47.5) & \\
\hline LVI & & & & 0.173 & & & & 0.264 \\
\hline Absent & 664 (70.8) & 343 (72.8) & $321(68.7)$ & & 733 (71.3) & 430 (72.8) & 303 (69.5) & \\
\hline Present & $274(29.2)$ & 128 (27.2) & 146 (31.3) & & 294 (18.7) & $161(27.2)$ & 133 (30.5) & \\
\hline LNM & & & & 0.242 & & & & 0.205 \\
\hline Absent & $257(27.4)$ & $137(29.1)$ & $120(25.7)$ & & 286 (27.8) & $174(29.4)$ & 112 (25.7) & \\
\hline Present & $681(72.6)$ & 334 (70.9) & 347 (74.3) & & 741 (72.2) & $417(70.6)$ & 324 (74.3) & \\
\hline Pathologic T stage & & & & 0.688 & & & & 0.148 \\
\hline Total & 937 & & & & 1,025 & & & \\
\hline $\mathrm{T} 2$ & 147 (15.7) & 75 (15.9) & 72 (15.5) & & 169 (16.5) & 110 (18.7) & 59 (13.5) & \\
\hline T3 & $344(36.7)$ & $172(84.1)$ & 172 (84.5) & & $369(36.0)$ & 205 (34.8) & $164(37.6)$ & \\
\hline $\mathrm{T} 4$ & $446(47.6)$ & $224(47.6)$ & $222(47.6)$ & & $487(47.5)$ & $274(46.5)$ & 213 (48.9) & \\
\hline p53 IHC & & & & 0.180 & & & & 0.014 \\
\hline Total & 915 & & & & 1,008 & & & \\
\hline Wild-type pattern & $298(32.6)$ & 158 (34.7) & $140(30.4)$ & & $324(32.1)$ & 204 (35.3) & 120 (27.9) & \\
\hline Mutant pattern & $617(67.4)$ & 297 (65.3) & $320(69.6)$ & & 684 (67.9) & 374 (64.7) & $310(72.1)$ & \\
\hline EBER-ISH & & & & 0.002 & & & & $<0.001$ \\
\hline Total & 915 & & & & 1,008 & & & \\
\hline Negative & 853 (93.2) & 436 (95.8) & $417(92.6)$ & & 941 (93.4) & 568 (98.3) & 373 (86.7) & \\
\hline Positive & 62 (6.8) & 19 (4.2) & $43(7.4)$ & & $67(6.6)$ & $10(1.7)$ & 57 (13.3) & \\
\hline MMR protein IHC & & & & $<0.001$ & & & & $<0.001$ \\
\hline Total & 915 & & & & 1,008 & & & \\
\hline MMR-proficient & 814 (89.0) & 423 (93.0) & 391 (85.0) & & $898(89.1)$ & 533 (92.2) & 365 (84.9) & \\
\hline MMR-deficient & 101 (11.0) & $32(7.0)$ & $69(15.0)$ & & $110(10.9)$ & 45 (7.8) & 65 (15.1) & \\
\hline
\end{tabular}

Data are presented as number (\%) or mean \pm SD.

CDX, caudal type homeobox; LVI, lymphovascular invasion; LNM, lymph node metastasis; IHC, immunohistochemistry; EBER-ISH, Epstein-Barr virus encoding RNA in situ hybridization; MMR, mismatch repair.

Table 2. Expression Status of CDX-1 and CDX-2 According to the Histologic-Molecular Classification of Advanced Gastric Cancers

\begin{tabular}{|c|c|c|c|c|}
\hline \multirow{2}{*}{ Variable } & \multicolumn{2}{|c|}{ CDX-1, No. (\%) } & \multicolumn{2}{|c|}{ CDX-2, No (\%) } \\
\hline & Cases & Positive & Cases & Positive \\
\hline Total & 915 & 455 & 1,008 & 578 \\
\hline \multicolumn{5}{|l|}{ Histologic-molecular classification 1} \\
\hline EBV-positive & $62(6.8)$ & $19(4.2)$ & $67(6.6)$ & $10(1.7)$ \\
\hline MMR-deficient & $101(11.0)$ & $32(7.0)$ & $110(10.9)$ & $45(7.8)$ \\
\hline EBV-/MMR-p/p53-mutant & 475 (51.9) & $254(55.8)$ & 529 (52.5) & 331 (57.3) \\
\hline EBV-/MMR-p/p53-intact & $277(30.3)$ & 150 (33.0) & $302(30.0)$ & 192 (33.2) \\
\hline \multicolumn{5}{|l|}{ Histologic-molecular classification 2} \\
\hline EBV-positive & $62(6.8)$ & $19(4.2)$ & $67(6.6)$ & $10(1.7)$ \\
\hline MMR-deficient & $101(11.0)$ & $32(7.0)$ & 110 (10.9) & 45 (7.8) \\
\hline EBV-/MMR-p/intestinal and mixed type & $357(39.0)$ & $168(36.9)$ & $391(38.8)$ & $260(45.0)$ \\
\hline EBV-/MMR-p/diffuse type & $395(43.2)$ & 236 (51.9) & $440(43.7)$ & $263(45.5)$ \\
\hline
\end{tabular}

CDX, caudal type homeobox; EBV, Epstein-Barr virus; MMR, mismatch repair; MMR-p, MMR-proficient. 


\section{Subgrouping of advanced GCs}

Using the data that we previously reported, ${ }^{18}$ all cases were divided into four subgroups using a so-called "histologic-molecular classification" based on findings retrieved from previous large sample-sized studies on GC using the same cohort based on the Lauren classification, EpsteinBarr virus encoding RNA in situ hybridization (EBER-ISH) results, MMR proteins, and p53 IHC results. ${ }^{18}$ The four subgroups were defined as follows: histologic-molecular classification 1 (HMC1) comprised (1) EBV-positive, EBER-ISH positive; (2) MMR-deficient, EBER-ISH negative and MMR-deficient (MMR-d); (3) p53-mutant type, EBER-ISH negative, MMR-proficient (MMR-p), p53 mutant pattern; and (4) p53-intact type, EBER-ISH negative, MMR-p, and p53-intact pattern. HMC2 based on Lauren classification, comprised (1) EBV-positive, EBER-ISH positive; (2) MMR-d, EBER-ISH negative and MMR-d; (3) intestinal type, EBER-ISH negative, MMR-p, and intestinal or mixed type; and (4) diffuse type, EBER-ISH negative, MMR-p, and diffuse type.

\section{Statistical analysis}

The clinical and pathological data were analyzed using IBM SPSS software, version 20.0 (IBM Corp. Armonk, NY, USA). The Pearson chi-square test was used to analyze correlations between clinicopathological variables and CDX expression patterns. Overall survival (OS) was defined as the time interval from surgery until the date of tumor-related death from any cause or date of the last follow-up. Disease-free survival (DFS) was defined as the interval from surgery to the date of recurrence or date of the last follow-up. Survival curves were estimated using the Kaplan-Meier method and a log-rank test. Univariate and multivariate analyses to estimate the independent prognostic significance of CDX expression were carried out using the Cox regression analysis. Statistical significance was defined as $\mathrm{p}<0.05$.

\section{RESULTS}

\section{CDX-1 and CDX-2 expression patterns}

Clinicopathologic characteristics according to CDX-1 and CDX-2 expression are summarized in Table 1, expression status of CDX-1 and CDX-2 according to the histomolecular classification is shown in Table 2, and representative photomicrographs of CDXs IHC staining are shown in Fig. 1. Because of a few dropouts of TMA cores in section process and omitted medical records of some patients, the numbers of clinicopathologic parameters were different in Table 1. Among the 938 cases with CDX-1 examination, CDX-1 was positive (1+ or $2+)$ in 471 cases $(50.2 \%)$ and negative in 467 cases (49.8\%). Among the 1,027 cases with CDX-2 examination, CDX-2 was positive (1+ or $2+)$ in 591 cases (57.5\%) and negative in 436 cases (42.5\%). Of the 915 GCs for CDX-1, 62 (6.8\%) were EBV-positive, 101 (11.0\%) were MMR-d, 475 (51.9\%) were p53-mutant, and the remaining 277 (30.3\%) were p53-intact type GCs. Of the 1,008 cases for CDX-2, 67 (6.6\%) were EBV-positive, 110 (10.9\%) were MMR-d, 529 (52.5\%) were p53-mutant, and the remaining $302(30.0 \%)$ were p53-intact type GCs.

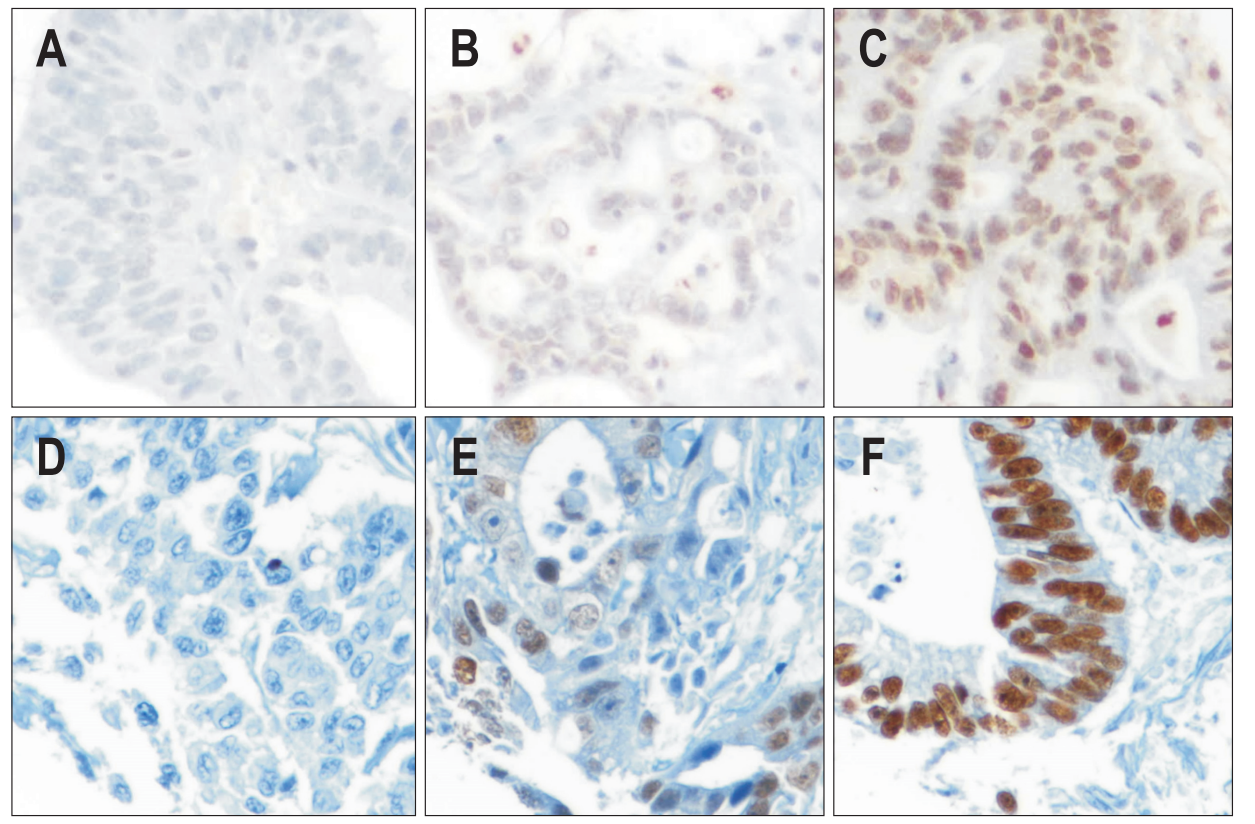

Fig. 1. Immunohistochemical staining for CDX-1 (A-C) and CDX-2 (D-F). Representative photomicrographs of negative, $1+$, and $2+$ cases of CDX-1 and CDX-2 expression ( $\times 400$ ). CDX, caudal type homeobox. 


\section{Survival analysis}

\section{1) Kaplan-Meier method}

The results of survival analysis using the Kaplan-Meier survival curves are summarized in Figs 2 and 3. Due to the omitted medical records of some patients, the numbers of patients subjected to the survival analysis were 902 for OS of CDX-1, 899 for DFS of CDX-1, 991 for OS of CDX2 and 986 for DFS of CDX-2. In EBV-positive or MMR-d GC groups, the Kaplan-Meier survival curves showed no significant differences according to CDX-1 or CDX-2 expression status in either DFS or OS (Figs $2 \mathrm{~B}$ and $\mathrm{C}, 3 \mathrm{~B}$ and C). In EBV-negative/MMR-p, p53-mutant, and p53-intact GCs, the expressions of CDX-1 and CDX-2 were associated with a significantly favorable OS $(\mathrm{p}=0.021, \mathrm{p}=0.047$ for CDX-1; $\mathrm{p}=0.045, \mathrm{p}=0.002$ for CDX-2, respectively) (Figs $2 \mathrm{D}$ and $\mathrm{E}, 3 \mathrm{D}$ and $\mathrm{E}$ ). In EBV-negative/MMR-p, intestinal-, and diffuse-type GCs, the expressions of CDX-1 and CDX2 were also correlated with a significantly favorable OS, regardless the histologic type $(\mathrm{p}=0.015, \mathrm{p}=0.019$ for CDX$1 ; \mathrm{p}=0.010, \mathrm{p}=0.042$ for $\mathrm{CDX}-2$, respectively) (Figs $2 \mathrm{~F}$ and $\mathrm{G}, 3 \mathrm{~F}$ and $\mathrm{G})$. Interestingly, in terms of DFS, the expression of CDX-1 was correlated with a better DFS only in p53mutant GCs ( $\mathrm{p}=0.011)$ by HMC1 and diffuse-type GCs $(\mathrm{p}=0.001)$ by HMC2 (Fig. 4). The expression of CDX-2 showed a correlation with a better DFS in both p53-mutant and $\mathrm{p} 53$-intact GCs ( $\mathrm{p}=0.032$ and $\mathrm{p}=0.016$, respectively); however, under HMC2, only diffuse-type GCs showed a significantly better prognosis ( $\mathrm{p}=0.037$ ) (Fig. 5).

\section{2) Univariate and multivariate analyses}

In EBV-positive and MMR-d GCs, univariate and multivariate survival analyses revealed no significant differences in DFS and OS according to CDX-1 and CDX-2 expression. In univariate analysis, in EBV-negative, MMRp GCs, both CDX-1 and CDX-2 expression was correlated with favorable OS ( $\mathrm{p}=0.003$ and $\mathrm{p}=0.001$, respectively). Moreover, CDX-1 and CDX-2 expression was correlated with favorable DFS in univariate analysis ( $\mathrm{p}=0.011$ and $\mathrm{p}=0.002$, respectively). In multivariate analysis, CDX -1 and CDX-2 expression showed independent prognostic significance in OS ( $\mathrm{p}=0.019$ and $\mathrm{p}=0.009$, respectively) and DFS $(\mathrm{p}=0.013$ and $\mathrm{p}=0.049$, respectively) (Table 3 ).

\section{DISCUSSION}

It is well known that CDX-2 is ectopically expressed in intestinal metaplasia in the stomach and that intestinal metaplasia is a precursor of intestinal-type gastric adenocarcinomas. ${ }^{19,20}$ In a mouse model, the gastric expression of CDX-2 alone was sufficient to induce intestinal metaplasia. ${ }^{1}$ In addition, induced intestinal metaplasia in the stomach of CDX-2-transgenic mice caused gastric adenocarcinoma. ${ }^{2}$ Therefore, CDX-2 has generally been recognized as a marker reflective of the progression of carcinogenesis from chronic gastritis-intestinal metaplasia to GC in humans. ${ }^{4,5}$ In terms of Lauren classification, the intestinal Lauren subtype may be associated with the expression of CDX-2 and its prognostic relationship. However, considering the results of this study, the proportion of patients expressing CDX-2 among diffuse-type GCs (47.7\%) was similar to that among intestinal-type GCs (52.4\%; $\mathrm{p}=0.095)$. Furthermore, a relationship between CDX-2 expression and favorable prognosis was found in intestinal- and diffuse-type GCs ( $\mathrm{p}=0.01 \mathrm{vs} \mathrm{p}=0.042$ ) (Fig. 3 ). These results suggest that $\mathrm{CDX}-2$ expression may affect the carcinogenesis of diffuse-type GCs or at least a subset thereof.

The prognostic significance of CDX-2 expression in GC has been controversial for a long time. In our results, the expression of both CDX-1 and CDX-2 was not related to a favorable prognosis in EBV-positive and in MMR-d GCs, and in the case of CDX-2, the tendency was even reversed. Only among EBV-negative and MMR-p GCs were CDX1 and CDX-2 independent favorable prognostic factors. These results suggest that the heterogeneity of GCs in molecular or histologic subtypes is very important and should be considered when designing a study mining and analyzing the prognostic factors of GC. Controversial results from other studies on CDX-2 in GC may have been caused by the lack of consideration of this molecular or histologic heterogeneity among GCs. Even in our study, the expression of CDX-2 was not statistically related to patient survival in the overall GC group.

In this study, we demonstrated that patient survival for CDX-1-positive GCs was better than that of CDX1-negative GCs. This result is supported by the results of our previous RNA-profiling-based study, in which CDX1 was a predictive prognostic factor of favorable patient survival after adjuvant chemotherapy. ${ }^{17}$ Similarly, CDX2 expression was also found to be a favorable prognostic marker in both intestinal- and diffuse-type GCs. However, in contrast with the results for CDX-2 in our data, CDX-1 expression tended to show good prognosis in cases of both EBV-positive and MMR-d GCs, and was also a statistically significant favorable prognostic factor in overall GC cases. Therefore, the expression of CDX-1 may be superior to CDX-2 for evaluating the prognosis of stomach cancer patients.

In addition to subgrouping by EBV, MMR status, and Lauren classification, we also performed a subgroup analysis according to p53 expression patterns, which is a well- 

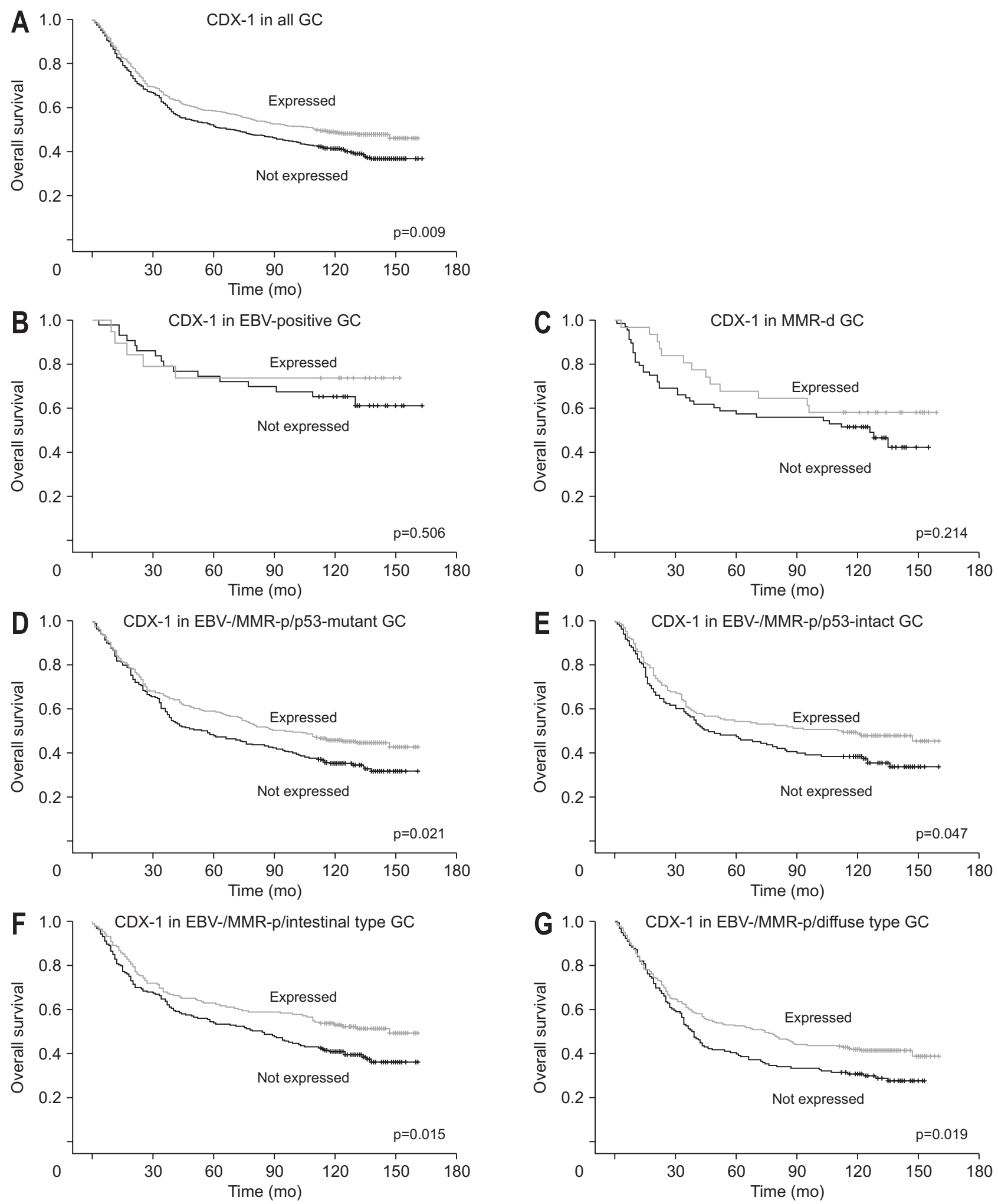

Fig. 2. Comparison of overall survival according to CDX-1 expression. (A) GC patients expressing CDX-1 showed a favorable prognosis. (B, C) There was no significant survival difference in both EBV-positive GC and MMR-d GC groups. (D, E) GC patients expressing CDX-1 showed a favorable prognosis among those with EBV-negative/MMR-p p53-mutant and p53-intact GCs. (F, G) GC patients expressing CDX-1 showed a favorable prognosis among those with EBV-negative/MMR-p, intestinal type and diffuse-type GCs.

CDX, caudal type homeobox; GC, gastric cancer; EBV, Epstein-Barr virus; MMR, mismatch repair; MMR-d, MMR-deficient; MMR-p, MMR-proficient. 

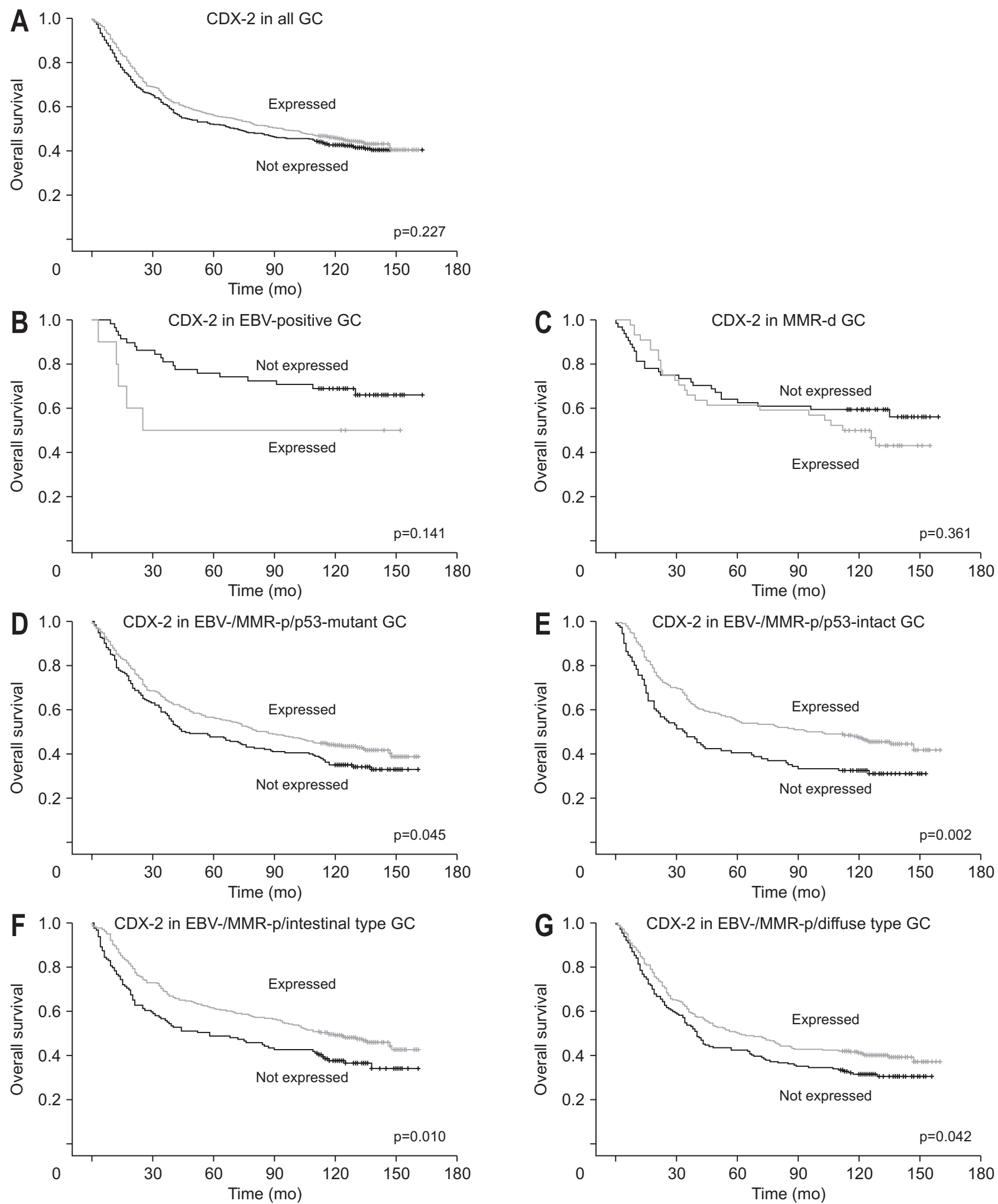

Fig. 3. Comparison of overall survival according to CDX-2 expression. (A) GC patients expressing CDX-2 showed a favorable prognosis. (B, C) There was no significant survival difference between the EBV-positive and MMR-d GC groups. (D, E) GC patients expressing CDX-2 showed a favorable prognosis among those with EBV-negative/MMR-p p53-mutant and p53-intact GCs. (F, G) GC patients expressing CDX-2 showed a favorable prognosis among those with EBV-negative/MMR-p, intestinal type and diffuse-type GCs.

CDX, caudal type homeobox; GC, gastric cancer; EBV, Epstein-Barr virus; MMR, mismatch repair; MMR-d, MMR-deficient; MMR-p, MMR-proficient. 

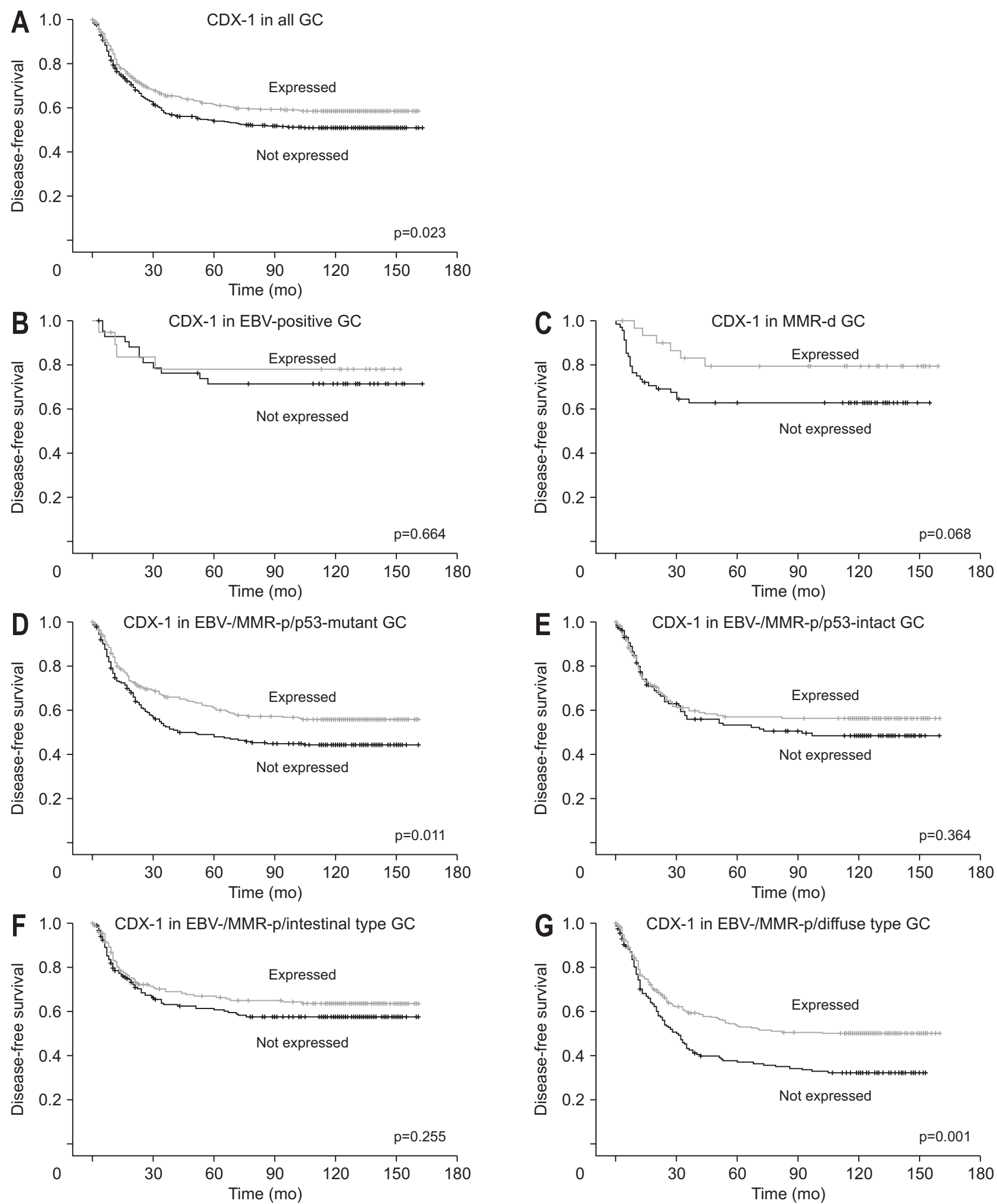

Fig. 4. Comparison of disease-free survival according to CDX-1 expression. (A) GC patients expressing CDX-1 showed a favorable prognosis. (B, C, E) There was no significant survival difference between the EBV-positive/MMR-d and EBV-negative/MMR-p p53-intact GC groups. (D) GC patients expressing CDX-1 showed a favorable prognosis among those with EBV-negative/MMR-p p53-mutant GC. (F) There was no significant survival difference between the EBV-negative and MMR-p intestinal type GC groups. (G) GC patients expressing CDX-1 showed a favorable prognosis among those with EBV-negative/MMR-p diffuse-type GC.

CDX, caudal type homeobox; GC, gastric cancer; EBV, Epstein-Barr virus; MMR, mismatch repair; MMR-d, MMR-deficient; MMR-p, MMR-proficient. 

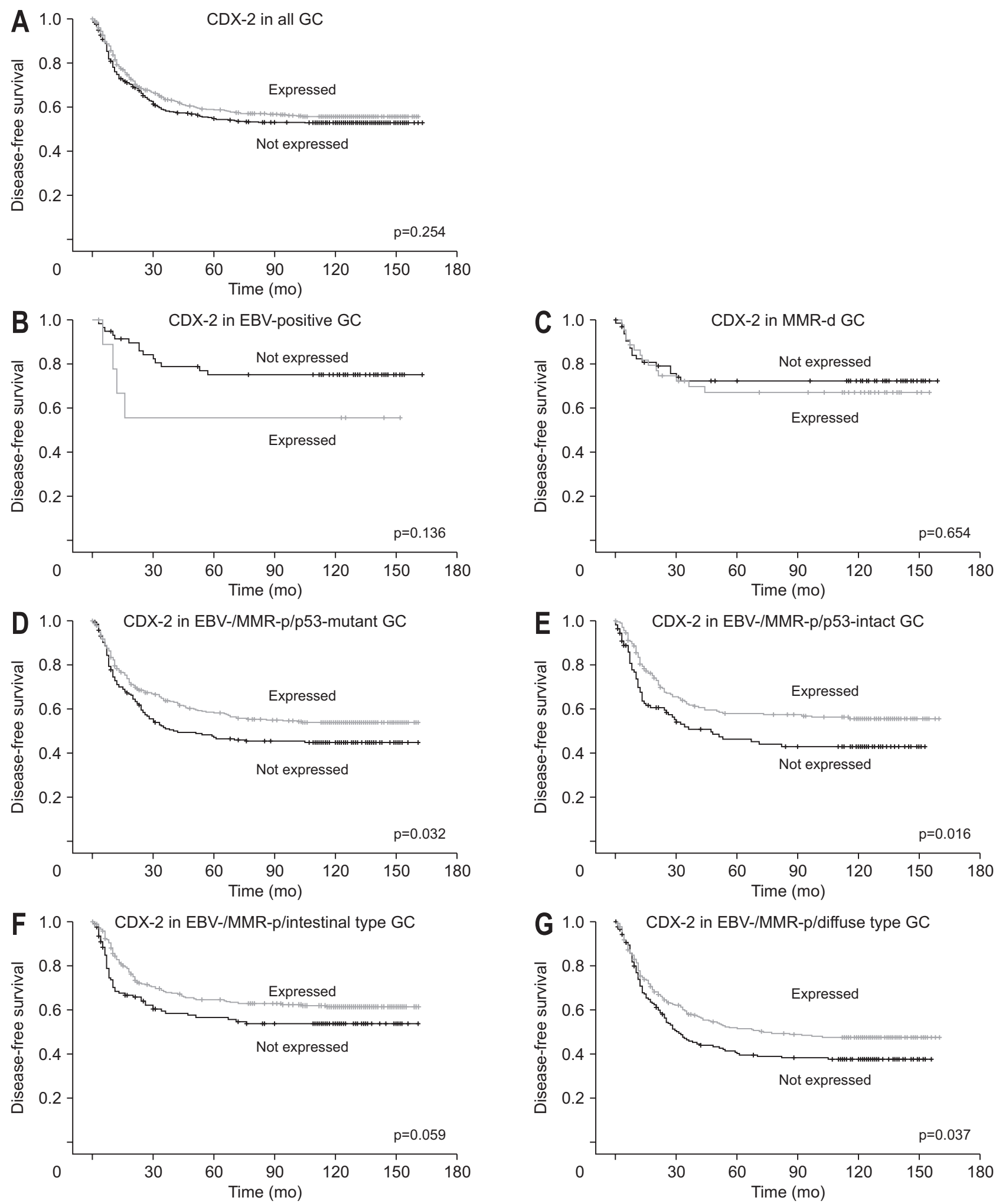

Fig. 5. Comparison of disease-free survival according to CDX-2 expression. (A) GC patients expressing CDX-2 showed no significant survival benefit. (B, C) There was no significant survival difference between the EBV-positive and MMR-d GC groups. (D, E) GC patients expressing CDX-2 showed a favorable prognosis among those with EBV-negative/MMR-p p53-mutant and p53-intact GCs. (F) There was no significant survival difference between the EBV-negative and MMR-p intestinal type GC groups. (G) GC patients expressing CDX-2 showed a favorable prognosis among those with EBV-negative/MMR-p diffuse-type GC.

CDX, caudal type homeobox; GC, gastric cancer; EBV, Epstein-Barr virus; MMR, mismatch repair; MMR-d, MMR-deficient; MMR-p, MMR-proficient. 
Table 3. Univariate and Multivariate Survival Analyses of Patients with EBV-Negative/MMR-Proficient Advanced Gastric Cancer

\begin{tabular}{|c|c|c|c|c|c|c|c|c|}
\hline \multirow{3}{*}{ Factor } & \multicolumn{4}{|c|}{ Overall survival } & \multicolumn{4}{|c|}{ Disease-free survival } \\
\hline & \multicolumn{2}{|l|}{ Univariate } & \multicolumn{2}{|l|}{ Multivariate } & \multicolumn{2}{|l|}{ Univariate } & \multicolumn{2}{|l|}{ Multivariate } \\
\hline & $\operatorname{HR}(95 \% \mathrm{CI})$ & $\mathrm{p}$-value & $\operatorname{HR}(95 \% \mathrm{Cl})$ & p-value & $\operatorname{HR}(95 \% \mathrm{CI})$ & $\mathrm{p}$-value & $\operatorname{HR}(95 \% \mathrm{Cl})$ & p-value \\
\hline Age & & $<0.001$ & & $<0.001$ & & 0.656 & & 0.636 \\
\hline$\leq 55 \mathrm{yr}$ & 1 & & 1 & & 1 & & 1 & \\
\hline$>55 \mathrm{yr}$ & $1.374(1.156-1.633)$ & & $1.547(1.258-1.901)$ & & 0.925 (0.762-1.123) & & $1.057(0.842-1.326)$ & \\
\hline Sex & & 0.099 & & 0.248 & & 0.036 & & 0.519 \\
\hline Male & 1 & & 1 & & 1 & & 1 & \\
\hline Female & $1.156(0.973-1.374)$ & & $1.128(0.920-1.384)$ & & 1.276 (1.047-1.555) & & 1.080 (0.855-1.365) & \\
\hline Differentiation & & $<0.001$ & & 0.220 & & $<0.001$ & & 0.373 \\
\hline Differentiated & 1 & & 1 & & 1 & & 1 & \\
\hline Undifferentiated & $1.536(1.262-1.869)$ & & $1.198(0.897-1.600)$ & & 1.759 (1.387-2.231) & & $1.175(0.824-1.676)$ & \\
\hline Lauren classification & & 0.008 & & 0.546 & & $<0.001$ & & 0.180 \\
\hline Intestinal or mixed & 1 & & 1 & & 1 & & 1 & \\
\hline Diffuse & 1.260 (1.063-1.493) & & $1.083(0.836-1.403)$ & & 1.539 (1.260-1.880) & & 1.235 (0.908-1.680) & \\
\hline Pathologic T stage & & $<0.001$ & & $<0.001$ & & $<0.001$ & & $<0.001$ \\
\hline $2 / 3$ & 1 & & 1 & & 1 & & 1 & \\
\hline 4 & 2.549 (2.142-3.035) & & $2.159(1.763-2.644)$ & & $3.124(2.541-3.841)$ & & $2.397(1.887-3.045)$ & \\
\hline LVI & & $<0.001$ & & 0.002 & & $<0.001$ & & 0.008 \\
\hline Absent & 1 & & 1 & & 1 & & 1 & \\
\hline Present & $2.167(1.818-2.584)$ & & $1.392(1.128-1.718)$ & & $2.210(1.805-2.706)$ & & 1.387 (1.090-1.763) & \\
\hline Lymph node metastas & & $<0.001$ & & $<0.001$ & & $<0.001$ & & $<0.001$ \\
\hline Absent & 1 & & 1 & & 1 & & 1 & \\
\hline Present & $3.243(2.591-4.061)$ & & $2.405(1.824-3.172)$ & & $3.800(2.887-5.001)$ & & $2.444(1.773-3.369)$ & \\
\hline CDX-1 & & 0.003 & & 0.019 & & 0.011 & & 0.013 \\
\hline Negative & 1 & & 1 & & 1 & & 1 & \\
\hline Expressed & $0.756(0.629-0.909)$ & & $0.780(0.635-0.960)$ & & $0.759(0.614-0.938)$ & & 0.741 (0.585-0.938) & \\
\hline CDX-2 & & 0.001 & & 0.009 & & 0.002 & & 0.049 \\
\hline Negative & 1 & & 1 & & 1 & & 1 & \\
\hline Expressed & $0.733(0.614-0.876)$ & & $0.759(0.618-0.932)$ & & $0.722(0.588-0.885)$ & & 0.789 (0.624-0.999) & \\
\hline
\end{tabular}

EBV, Epstein-Barr virus; MMR, mismatch repair; HR, hazard ratio; Cl, confidence interval; LVI, lymphovascular invasion; CDX, caudal type homeobox.

known surrogate marker for p53 mutation status. In addition to the TCGA (The Cancer Genome Atlas) molecular subtype, by which GC is divided into EBV-positive, MMR$\mathrm{d}$, genomically stable, and chromosomally unstable groups, another well-known molecular GC subgroup is the socalled Asian Cancer Research Group classification, which is based on expression profiling and principal component analysis. In the Asian Cancer Research Group classification, GCs are divided into MMR-d, MMR-p, epithelial-tomesenchymal transition, TP53-active, and TP53-inactive types. ${ }^{21}$ Therefore, we used the IHC $\mathrm{p} 53$ expression pattern as a marker for TP53-active and -inactive types. In our study, CDX-1 and CDX-2 expression was correlated with a favorable prognosis regardless of p53 expression pattern in the EBV-negative and MMR-p groups. Therefore, we reaffirmed that CDX-1 and CDX-2 could be reliable prognostic markers in EBV-negative and MMR-p GCs.

In terms of the limitations of this study, our cohort excluded patients who had previously undergone preoperative chemo- or radiotherapy, as well as patients who had undergone surgery for recurrent cancer. However, patients who received adjuvant chemotherapy were not excluded. Although CDX-1 and CDX-2 expression was statistically significant as a prognostic factor, postoperative therapeutic effects may be a confounding factor in patient survival.

In conclusion, our large study demonstrated that CDXpositive GCs are correlated with superior patient survival, compared to CDX-negative GCs among EBV-negative, MMR-p GCs. Furthermore, the prognostic value of CDX positivity was also relevant in diffuse-type GCs, not just to intestinal- and mixed-type GCs.

\section{CONFLICTS OF INTEREST}

No potential conflict of interest relevant to this article was reported. 


\section{ACKNOWLEDGEMENTS}

This work was supported by a National Research Foundation of Korea (NRF) grant funded by the Korea government (MSIT) (2019R1A2C1011355).

\section{AUTHOR CONTRIBUTIONS}

Study concept and design: H.K. Data acquisition: K.K., S.N. Data analysis and interpretation: H.K., K.K. Drafting of the manuscript: H.K., K.K. Critical revision of the manuscript for important intellectual content: H.K., J.H.C. Statistical analysis: K.K. Obtained funding: H.K. Study supervision: H.K.

\section{ORCID}

Kyeongmin Kim https://orcid.org/0000-0002-0454-4393 Songmi Noh https://orcid.org/0000-0001-9711-9996 Jae-Ho Cheong https://orcid.org/0000-0002-1703-1781 Hyunki Kim https://orcid.org/0000-0003-2292-5584

\section{REFERENCES}

1. Silberg DG, Sullivan J, Kang E, et al. Cdx2 ectopic expression induces gastric intestinal metaplasia in transgenic mice. Gastroenterology 2002;122:689-696.

2. Mutoh H, Sakurai S, Satoh K, et al. Cdx1 induced intestinal metaplasia in the transgenic mouse stomach: comparative study with Cdx2 transgenic mice. Gut 2004;53:1416-1423.

3. Lee BH, Kim N, Lee HS, et al. The role of CDX2 in intestinal metaplasia evaluated using immunohistochemistry. Gut Liver 2012;6:71-77.

4. Wang XT, Wei WY, Kong FB, et al. Prognostic significance of Cdx2 immunohistochemical expression in gastric cancer: a meta-analysis of published literatures. J Exp Clin Cancer Res 2012;31:98

5. Choi SI, Yoon C, Park MR, et al. CDX1 expression induced by CagA-expressing Helicobacter pylori promotes gastric tumorigenesis. Mol Cancer Res 2019;17:2169-2183.

6. Almeida R, Silva E, Santos-Silva F, et al. Expression of intestine-specific transcription factors, CDX1 and CDX2, in intestinal metaplasia and gastric carcinomas. J Pathol 2003;199:36-40.

7. Park DY, Srivastava A, Kim GH, et al. CDX2 expression in the intestinal-type gastric epithelial neoplasia: frequency and significance. Mod Pathol 2010;23:54-61.

8. Xie Y, Li L, Wang X, et al. Overexpression of Cdx2 inhibits progression of gastric cancer in vitro. Int J Oncol 2010;36:509-516.

9. Qin R, Wang NN, Chu J, Wang X. Expression and significance of homeodomain protein $\mathrm{Cdx} 2$ in gastric carcinoma and precancerous lesions. World J Gastroenterol 2012;18:3296-3302.

10. Ru Y, Zhang L, Chen Q, et al. Detection and clinical significance of lymph node micrometastasis in gastric cardia adenocarcinoma. J Int Med Res 2012;40:293-299.

11. Ge J, Chen Z, Wu S, Yuan W, Hu B, Chen Z. A clinicopathological study on the expression of cadherin-17 and caudalrelated homeobox transcription factor (CDX2) in human gastric carcinoma. Clin Oncol (R Coll Radiol) 2008;20:275283.

12. Roessler K, Mönig SP, Schneider PM, et al. Co-expression of CDX2 and MUC2 in gastric carcinomas: correlations with clinico-pathological parameters and prognosis. World J Gastroenterol 2005;11:3182-3188.

13. Xiao ZY, Ru Y, Sun JT, et al. Expression of CDX2 and villin in gastric cardiac intestinal metaplasia and the relation with gastric cardiac carcinogenesis. Asian Pac J Cancer Prev 2012;13:247-250.

14. Youn YH, Byun HJ, Yoon JH, Park CH, Lee SK. Long noncoding RNA N-BLR upregulates the migration and invasion of gastric adenocarcinoma. Gut Liver 2019;13:421-429.

15. Bae YS, Kim H, Noh SH, Kim H. Usefulness of immunohistochemistry for microsatellite instability screening in gastric cancer. Gut Liver 2015;9:629-635.

16. Cancer Genome Atlas Research Network. Comprehensive molecular characterization of gastric adenocarcinoma. Nature 2014;513:202-209.

17. Cheong JH, Yang HK, Kim H, et al. Predictive test for chemotherapy response in resectable gastric cancer: a multicohort, retrospective analysis. Lancet Oncol 2018;19:629638 .

18. Park CK, Park JS, Kim HS, et al. Receptor tyrosine kinase amplified gastric cancer: clinicopathologic characteristics and proposed screening algorithm. Oncotarget 2016;7:72099-72112.

19. Huang RJ, Choi AY, Truong CD, Yeh MM, Hwang JH. Diagnosis and management of gastric intestinal metaplasia: current status and future directions. Gut Liver 2019;13:596-603.

20. Hwang JH. Understanding gastric cancer risk factors: we need to close the gap. Gut Liver 2018;12:1-2.

21. Cristescu R, Lee J, Nebozhyn M, et al. Molecular analysis of gastric cancer identifies subtypes associated with distinct clinical outcomes. Nat Med 2015;21:449-456. 